\title{
Developments of X-ray Fluorescence Tomography at 2-ID-E at APS for Studies of Composite Samples
}

\author{
$\underline{\text { Olga Antipova }}{ }^{1}$, Kenneth Kemner $^{2}$, Christian Roehrig ${ }^{1},{\text { Stefan } \operatorname{Vogt}^{1}, \mathrm{Lu} \mathrm{Xi} \mathrm{Li}^{1} \text {, and Doga Gursoy }}^{1}$ \\ 1. X-ray Sciences Division, Argonne National Laboratory, Argonne, IL, USA. \\ 2. Biosciences Division, Argonne National Laboratory, Argonne, IL, USA. \\ * Corresponding author, oantipova@anl.gov.
}

The X-ray Fluorescence (XRF) is one of the most important tools for elemental quantification and mapping in biological and medical samples[1]. Our recent efforts has been focused on expansion of regular two-dimensional X-ray Fluorescence imaging in order to obtain three-dimensional distribution of various elements within relatively large sample without sectioning, which may result in sample damages and artifacts. We have improved XRF set up and tested different data acquisition schemes for optimal tomographic reconstructions using TomoPy [2].

Here we present our progress in 3D visualization of rather large and dense sample of soil with distribution Selenium-labeled bacteria through it. Optimizations developed for this study are crucial for biological, environmental sciences and material science applications.

Soil is a great example of challenging sample to study with X-ray fluorescence tomography due to its heterogeneity, opacity, diversity of mineral and organic components of varying sizes, pores filled with air, liquids or microorganisms. It provides growing medium for bacteria, plants, and fungi, which are involved in large variety of biogeochemical functions, such as carbon fixation, nitrogen cycling, metal binding and remediation of certain pollutants. Therefore, understanding soil microscopic structure and composition and its role in bacterial migration and function is crucial for large number of environmental sciences applications.

In order to map bacterial migration through soil specimen in three dimensions and at different time intervals, Pseudomonas protogens were labeled with quantum dots. Cadmium-selenium(core) and zincsulfur (shell) quantum dot, functionalized with glycine to ensure uptake by bacteria [3], can be imaged within microorganisms using both X-ray fluorescence mapping and visible light microscopy. Then 0.5-1 $\mathrm{mm}^{3}$ moist soil aggregates were incubated with quantum dot-labeled bacteria for 5 and 15 days and ten fixed and embedded in paraffin to prevent sample fragmentation due to dehydration. Sectioning of this sample causes significant sample degradation and soil opacity limits imaging techniques to X-ray transmission and fluorescence tomography in order to resolve three-dimensional pore and granules soil structure and map bacterial penetration trough it in course of five and fifteen days.

X-ray fluorescence mapping in 2D and 3D (Figure 1 and 2) was performed at 2-ID-E beamline and X-ray transmission tomography data was collected at 2-BM beamline (Figure 2) at the Advanced Photon Source (Argonne National Laboratory). While transmission data provided enough information about 3D structure of soil aggregate incubated with Selenium-labeled bacteria for five days, it did not provide contrast to trace bacteria themselves. Therefore, we rely on X-ray fluorescence data to trace bacteria and correlate it with transmission data.

Soil aggregate was pre-scanned at 2-ID-E to obtain initial 2D map with $0.5 \times 0.5$ resolution and 100 
milliseconds dwell time using zone plate-based optics and four-element Vortex detector at $13.7 \mathrm{keV}$ (Figure 1). This image was used to select $30 \mu \mathrm{m}$ slice for tomography scan. Initial approach with $180^{\circ}$ angular span and 60 projections with $1 \times 1 \mu \mathrm{m}$ pixel size and $100 \mathrm{msec}$ dwell time resulted in multiple reconstruction artifacts due to high self-absorbtion of soil components. Therefore, new approach was used to minimize self-absorption effects. Approximatley $600 \mu \mathrm{m}$ wide and $5 \mu \mathrm{m}$ tall slice was scanned to obtain 600 projection with $1 \times 1 \mu \mathrm{m}$ pixel size and $100 \mathrm{msec}$ dwell time to get strong enough signal from Selenium. $\mathrm{X}$-Ray fluorescence data was collected as four interlaced sets of 150 projections each resulting in $0.3^{\circ}$ angular separation between projections in whole set with $180^{\circ}$ angular span of whole data set.

TomoPy was used for 3D reconstruction with Penalized Maximum Likelihoods, which helped to significantly reduce self-absorption artifacts and obtain map of Selenium distribution on surface and inside the soil sample within $5 \mu \mathrm{m}$ slice (Figure 2), where Selenium indicates bacteria labeled with CadmiumSelenium quantum dots. In addition slice reconstructed from X-ray fluorescence tomography data was matched with X-ray transmission data collected at 2-BM.

Self-absorption is a great challenge to face in soil studies and it was solved by increasing amount of projection for one data set. However, it is very time-consuming and also suffers from beam fluctuations. Future APS upgrade will provide increase in flux to enable us to run these studies routinely to explore more conditions.

\section{References:}

[1] D. Bourassa, et al, Metallomics 6.9 (2014) 1648 - MAPS and zebrafish

[2] D. Gursoy et al, J. of Synchrot. Rad. 21.5 (2014) 1188 - TomoPy

[3] X. Michalet et al, Science 307 (2005) 538. Q-dots

[4]Use of the Advanced Photon Source, Office of Science user facilities, was supported by the U.S. Department of Energy, Office of Science, Office of Basic Energy Sciences, under Contract No. DE-AC0206CH11357.

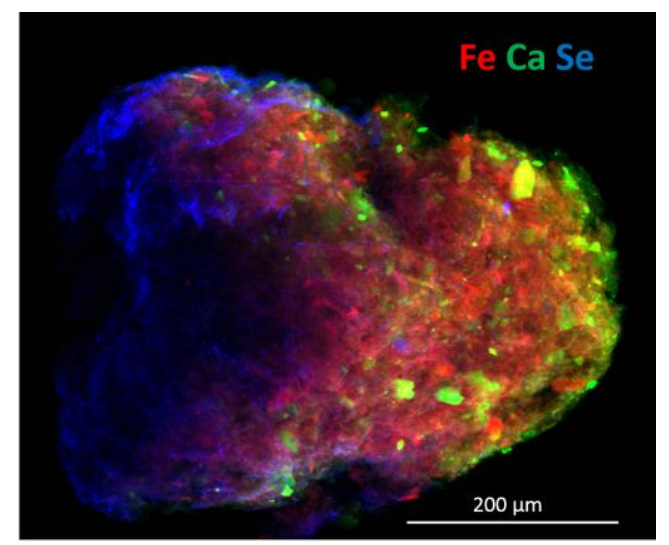

Figure. 1. X-ray fluorescence image of soil sample with Cadmium-selenium quantum dots labeled bacteria. $0.5 \times 0.5 \mu \mathrm{m}$ pixel 0.1 seconds dwell time
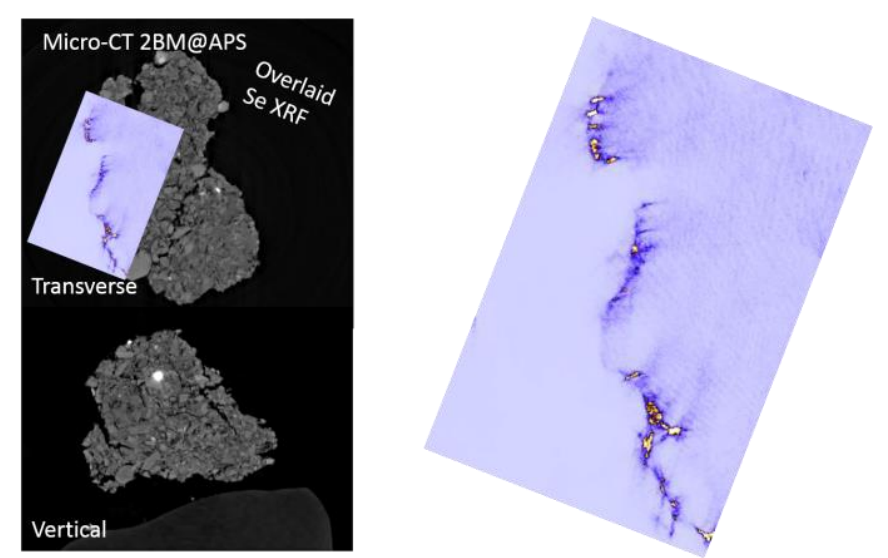

Figure. 2. X-ray transmission (left) and X-ray fluorescence (right) tomography reconstruction of matching slices of soil sample with Cadmiumselenium quantum dots labeled bacteria. 